Trauma Berufskrankh 2004 - 6 [Suppl 3] : S321-S323 DOI 10.1007/s10039-003-0766-4

Online publiziert: 26. August 2003

(c) Springer-Verlag 2003

Petra Gorschlüter · AOK Rheinland, Düsseldorf

\title{
Versorgungssicherung unter DRG-Bedingungen
}

\section{Verbesserte Qualität durch die DRG-Einführung}

Das DRG-System wird zu einem Wettbewerb um den Patienten führen. Diese $\mathrm{Pa}$ tientenorientierung äußert sich z. B. in einer kürzeren Verweildauer, denn welcher Patient will schon länger als unbedingt notwendig im Krankenhaus liegen. Gleichzeitig führt der Wettbewerb aber auch dazu, dass es sich ein Krankenhaus nicht leisten kann, Patienten aus Kostengründen zu früh zu entlassen. Eine stärkere Transparenz ermöglicht es Patienten, sich vor einem Krankenhausaufenthalt umfassend zu informieren. Zu diesem Zweck sollen die ab 2004 zu erstellenden Qualitätsberichte (u. a. im Internet) veröffentlicht werden. Diese Regelung sowie die Vorgabe von Mindestmengen für planbare Leistungen zeigt, dass die Einführung der DRG auch mit der Zielsetzung einer stärkeren Spezialisierung der Krankenhäuser verbunden ist.

\section{Sicherstellung der adäquaten Finanzierung}

Im Fallpauschalengesetz sind genügend Sicherheitsnetze ausgelegt worden, damit alle medizinisch notwendigen Leistungen auch adäquat finanziert werden. Dazu gehört eine Öffnungsklausel, die sicherstellt, dass Leistungen, für die in der Erstversion 2003/2004 noch keine Fallpauschalen gebildet werden konnten, durch Vereinbarungen vor Ort vergütet werden können. Diese Regelung wird jetzt durch das Fallpauschalenänderungsgesetz (FPÄndG) bis 2005/2006 verlängert und darüber hinaus inhaltlich sogar erweitert.
Es greift auch eine Grenzverweildauerregelung, die bei Fällen, die aufgrund der Schwere der Krankheit überdurchschnittlich lange liegen, wieder tagesbezogene Zuschläge vorsieht. Diese Regelung dient dazu, das Risiko von „Ausreißerfällen“ für die Krankenhäuser zu begrenzen und eine adäquate Behandlung sicherzustellen.

\section{Folgen der DRG-Einführung}

Mit der Einführung der DRG werden sich bestimmte Auswirkungen zeigen:

- Verkürzung der Verweildauer im Krankenhaus

Durch die Einführung der DRG wird eine deutliche Verkürzung der Liegezeiten auftreten, was bereits jetzt an den ersten Umsteigerhäusern sichtbar wird. Allerdings wurden nach der Bundespflegesatzverordnung bereits 20-30\% der Fälle über Fallpauschalen abgerechnet. Bei diesen - vorwiegend chirurgischen - Fällen sind kaum weitere Kürzungen zu erwarten.

Die Verweildauerkürzungen sind v. a. durch verbesserte Organisationsstrukturen und geplante Abläufe zu erreichen. In diesem Zusammenhang spielt der Einsatz von Behandlungsleitfäden (clinical pathways) eine zentrale Rolle. Die so genannten „blutigen“ Entlassungen kann sich ein Krankenhaus im Wettbewerb nicht leisten, aber auch gesetzlich ist festgelegt, dass eine vorzeitige Verlegung oder Entlassung aus wirtschaftlichen Gründen unterbleiben (s. \$17c Abs. 1 Nr. 2 KHG). Die Einhaltung dieser Verpflichtung kann über nisation im Krankenhaus liegen die größten Rationalisierungspotenziale. 
Trauma Berufskrankh 2004 - 6 [Suppl 3] : S321-S323

DOI 10.1007/s10039-003-0766-4

C) Springer-Verlag 2003

\section{P.Gorschlüter}

\section{Versorgungssicherung unter DRG-Bedingungen}

\section{Zusammenfassung}

Ziele der DRG-Einführung sind mehr Leistungsgerechtigkeit, mehr Leistungstransparenz, mehr Wirtschaftlichkeit und Qualität. Das DRG-System wird zu einem Wettbewerb um den Patienten, der sich aufgrund der stärkeren Transparenz umfassender informieren kann, führen und damit zu einer besseren Versorgung. Auch eine stärkere Spezialisierung der Krankenhäuser wird eine positive Folge sein. Im Fallpauschalengesetz, im Fallpauschalenänderungsgesetz sowie der Grenzverweildauerregelung ist die adäquate Finanzierung notwendiger Leistungen gesichert. Die ersten Erfahrungen mit dem DRG-Umstieg wurden mit den Optionshäusern gewonnen. Erwartungsgemäß ist die Überprüfung der Kodierung ein

\section{Safeguarding care with the DRG system}

\begin{abstract}
The aims of introducing the DRG system are fairer remuneration based on services provided, enhanced transparency in terms of performance, better value for money, and a better quality of care. The DRG system will lead to competition for patients, who are able to find out more thanks to the greater transparency, and thus in turn to better care. Another positive effect will be the more marked specialization of hospitals. The laws on per-case lump sum payments and on alteration of the per-case lump sum payments and also the regulation governing hospital stay thresholds will ensure adequate financial provision for the services needed. Early experience with the changeover to the DRG-System has been recorded in clinics that opted to join in 2003 before it becomes compulsory. As might have been expected, the review of the coding system is a cen-
\end{abstract}

zentrales Problem und wird auch in Zukunft ein großes Aufgabenfeld bleiben. Einen Schwachpunkt bei der Einführung der DRGs im Optionsjahr stellt der DRG-Katalog 2003 dar. Dessen Weiterentwicklung hängt maßgeblich von der Beteiligung der Krankenhäuser und medizinischen Fachgesellschaften ab. Ein verbesserter DRG-Katalog sowie erweiterte Öffnungsklauseln sollten für 2004 eine gute Grundlage für den flächendeckenden Umstieg auf die DRG sein.

\section{Schlüsselwörter}

Diagnoseorientierte Fallpauschalen .

Krankenhaus · Krankenkasse · DRG-Einführung
Stichprobenprüfungen vom Medizinischen Dienst der Krankenkassen überprüft werden.

- Verschiebung der Patienten in andere Bereich bzw. der Drehtüreffekt Die Verschiebung von Leistungen aus dem Krankenhaus in den ambulanten Bereich ist - nach dem Grundsatz „ambulant vor stationär“ - ausdrücklich vom Gesetzgeber so gewollt. Bei der Verkürzung der Verweildauern ist es wichtig, dass die entsprechenden ambulanten und stationären Nachsorgestrukturen insbesondere in der Pflege und in der Rehabilitation zur Verfügung stehen. Hier ist v. a. ein verbessertes Schnittstellenmanagement von Seiten des Krankenhauses gefragt. Wird ein Patient zu früh entlassen, tritt der so genannte Drehtüreffekt bei einer Wiedereinweisung auf. Dem soll eine weitere Grenzverweildauer (GVD)-Regel entgegen wirken: Wird ein Patient innerhalb der GVD aufgrund derselben Ursache wieder eingeliefert, kann keine neue DRG abgerechnet werden.

- Spezialisierung der Krankenhäuser Im zukünftigen DRG-System ist es für Krankenhäuser entscheidend, eine hohe Fallzahl in einer Fallgruppe zu erreichen, um die so genannten Skalen- und Erfahrungskurveneffekte realisieren zu können. Das heißt, eine hohe Fallzahl wirkt sich zum einen positiv auf die medizinische Qualität aus und zum anderen sinken die durchschnittlichen Fallkosten durch den (Fixkosten-)Degressionseffekt. Die strategische Positionierung von Krankenhäusern wird daher zukünftig stark auf eine Spezialisierung ausgerichtet sein. Auch diese Entwicklung ist vom Gesetzgeber ausdrücklich so gewollt. Die Gefahr besteht darin, dass Krankenhäuser anfangen könnten, eine Patientenselektion zu betreiben. Dem steht aber die gesetzliche Verpflichtung zur Erfüllung ihres Versorgungsauftrags entgegen.

\section{Erste Erfahrungen}

Die ersten Erfahrungen mit dem DRGUmstieg wurden mit den Optionshäusern 
gewonnen. Am Anfang steht eine neue Art von Budgetverhandlungen, die v. a. um den Aspekt einer Leistungsplanung auf Basis von DRG erweitert werden. Als Ergebnis der Budgetverhandlungen wird der Basisfallwert des Krankenhauses vereinbart, der als „Preis“ für die DRG-Leistungen abgerechnet wird. Für eine reibungslose Abrechnung ist ein funktionsfähiger Datenaustausch zwischen Krankenhaus und Krankenkassen von zentraler Bedeutung. Bei der Vielzahl von Informationen, die zur Ermittlung der richtigen DRG notwendig sind, bedeutet es für beide Seiten einen unverhältnismäßig hohen Aufwand, wenn die DRG-Rechnungen noch auf Papier erfolgen.

Erwartungsgemäß ist die Überprüfung der Kodierung ein zentrales Problem. Es bestehen noch große Unsicherheiten, wie die Kodierrichtlinien im Einzelnen auszulegen sind. Zu diesem Zweck führen wir mit den Krankenhäusern Gespräche über Kodierauffälligkeiten, das wird auch in Zukunft ein großes Aufgabenfeld bleiben.

Einen Schwachpunkt bei der Einführung der DRG im Optionsjahr stellt der DRG-Katalog 2003 dar, der überwiegend auf der Basis des australischen Katalogs unter starkem Zeitdruck entstanden ist. Sowohl in der Differenzierung der Klassifikation als auch bei den Bewertungsrelationen besteht erheblicher Verbesserungsbedarf für 2004.

\section{Resümee}

Die Weiterentwicklung des DRG-Katalogs hängt maßgeblich von der Beteiligung der Krankenhäuser und medizinischen Fachgesellschaften am Vorschlagsverfahren für die DRG-Klassifikation und am Kalkulationsverfahren für die Neuberechnung der Relativgewichte ab. Ein verbesserter DRGKatalog sowie erweiterte Öffnungsklauseln (s. FPÄndG) sollten für 2004 eine gute Grundlage für den flächendeckenden Umstieg auf die DRG sein.

\section{Korrespondierender Autor Dr. Petra Gorschlüter}

AOK Rheinland,

Kasernenstraße 61,40213 Düsseldorf, E-Mail: petra.gorschlueter@rla.aok.de
„Trauma und Berufskrankheit" bietet aktuelle Beiträge zu Fragen der Unfall- und Wiederherstellungschirurgie, der Berufserkrankungen sowie zu Themen der Unfallverhütung, der operativen und konservativen Therapie.

Möchten Sie ein bereits erschienenes Heft nachbestellen? Die folgenden Ausgaben können Sie direkt bei unserem Kundenservice zum Preis von je EUR 35,- beziehen:

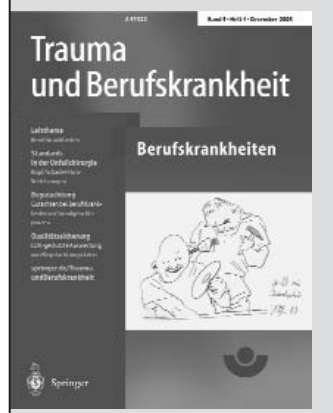

\section{Heft 4/2003}

Leitthema:

- Berufskrankheiten

Standards in der Unfallchirurgie:

- Kopf/Schädel-Hirn-Verletzungen

Begutachtung:

- Technische Gutachten im Sozialgerichtsprozess unter Berücksichtigung der Berufskrankheiten

Qualitätssicherung:

- EDV-gestützte Auswertung von Begutachtungsdaten

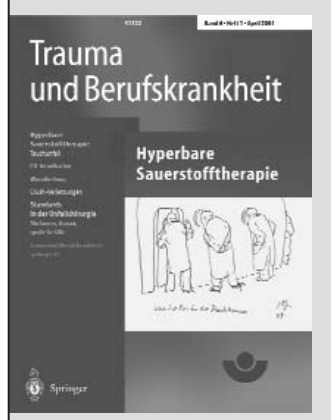

Heft 1/2004

Leitthema:

- Hyperbare Sauerstofftherapie

Standards in der Unfallchirurgie:

- Abdomen, Thorax, große Gefäße

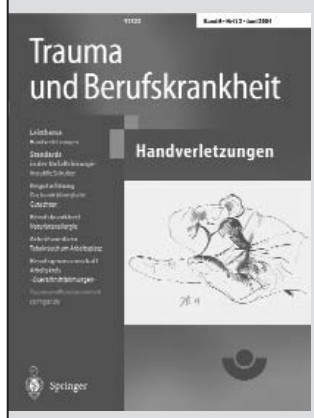

\section{Heft 2/2004}

Leitthema:

- Handverletzungen

Standards in der Unfallchirurgie:

- Instabile Schulter

Begutachtung:

- Das handchirurgische Gutachten

Berufskrankheit:

- Naturlatexallergie

Arbeitsmedizin:

- Tabakrauch am Arbeitsplatz

Begutachtung:

- Arbeitskreis „Querschnittlähmungen“

So erreichen Sie unseren Kundenservice:

Springer-Verlag

Kundenservice Zeitschriften

Haberstr. 7, 69126 Heidelberg

Tel.: +496221345-4303

Fax: +49 6221 345-4229

E-Mail:SAG-journals@springer-sbm.com

TraumaundBerufskrankheit.springer.de 